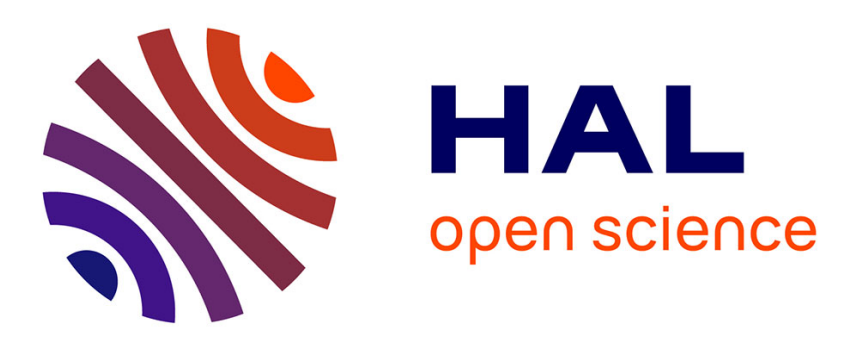

\title{
Des poissons aux masses d'eau: les usages militants du droit pour faire entendre la parole d'êtres qui ne parlent pas \\ C. Gramaglia
}

\section{- To cite this version:}

C. Gramaglia. Des poissons aux masses d'eau: les usages militants du droit pour faire entendre la parole d'êtres qui ne parlent pas. Politix, 2008, 21 (83), p. 133 - p. 153. hal-00472993

\section{HAL Id: hal-00472993 \\ https://hal.science/hal-00472993}

Submitted on 13 Apr 2010

HAL is a multi-disciplinary open access archive for the deposit and dissemination of scientific research documents, whether they are published or not. The documents may come from teaching and research institutions in France or abroad, or from public or private research centers.
L'archive ouverte pluridisciplinaire $\mathbf{H A L}$, est destinée au dépôt et à la diffusion de documents scientifiques de niveau recherche, publiés ou non, émanant des établissements d'enseignement et de recherche français ou étrangers, des laboratoires publics ou privés. 


\section{Des poissons aux masses d'eau : les usages militants du droit pour faire entendre la parole d'êtres qui ne parlent pas.}

\section{Christelle Gramaglia (Cemagref - Montpellier)}

Résumé : Cet article relate les efforts entrepris par l'association ANPER-TOS depuis 1958 pour dénoncer et faire réprimer les pollutions aquatiques. Il montre comment certains pêcheurs, aidés par des juristes, sont devenus les porte-parole d'entités non-humaines jusquelà exclues de la représentation. Il pose le problème de leur prise en charge politique étant donné leurs singularités et les médiations techniques souvent lourdes qu'elles requièrent avant de pouvoir être entendues. L'article évoque plus particulièrement une affaire de porcherie industrielle survenue en Lozère qui pose la question de la matérialité et des êtres à prendre en compte pour la composition des mondes ruraux communs.

Summary: This article accounts for the efforts made by ANPER-TOS since 1958 to blow the whistle and get water pollution acts sanctioned. It shows how some fishermen, helped by legal professionals, became the spoke-persons for non-human entities which were until then excluded from representation. It discusses the problem of their political acknowledgement and management considering their singularities and the highly technical mediations they need to be heard. The article evokes particularly a trial concerning a pig factory farming located in Lozère, in France, and questions the materiality and the number of entities that should be taken into account in the making of common rural worlds.

Contrairement à ce que l'on pourrait penser du fait de la multiplication des déclarations en faveur de l'environnement, le nombre des adhésions dans les associations françaises de protection de la nature n'a pas varié depuis vingt ans. Rassemblant une moyenne de 2 à $3 \%$ de la population, ces associations étaient environ trois mille en 2002 à s'être affiliées à la fédération nationale France-Nature-Environnement (FNE) ${ }^{1}$. Toutefois, les cadres et les formes des mobilisations environnementales ont changé en même temps que la législation évoluait. Après la création d'un ministère spécifique en 1971, les lois de 1976 et 1977 ont accompagné la prise en charge institutionnelle des questions environnementales, dans leur grande diversité $e^{2}$. Ces lois ont explicitement désigné les associations de protection de la nature comme relais de l'État sur le terrain pour la défense des intérêts qu'elles identifiaient, principalement la sauvegarde de la faune, de la flore et des espaces «naturels ». Celles-ci se sont alors vues dotées de moyens d'action particuliers, de manière à prolonger le travail engagé par le ministère de l'environnement dépourvu jusqu'alors de services

\footnotetext{
${ }^{1}$ Voir un ouvrage qui dresse le bilan de plusieurs années d'enquête : Boyer, (M.), Herzlich, (G.), Maresca, (B.), (dir.) L'environnement, question sociale. Dix ans de recherches pour le ministère de l'environnement, Odile Jacob, Paris, 2001.

2 Charvolin, (F.), L'invention de l'environnement en France. Chroniques anthropologiques d'une institutionnalisation, La Découverte, Paris, 2003. L'auteur a montré que la création du Ministère de l'environnement avait été rendue possible par la mise en rapport de domaines séparés: les pollutions, les nuisances, la conservation des espaces naturels et une partie de l'aménagement du territoire. La nébuleuse des associations de protection de la nature, pour reprendre une expression employée par André Micoud pour qualifier le tissu à la fois dense et très hétérogène qu'elles constituent, réunit des amateurs et des militants qui ont, pour la plupart, développé des compétences expertes dans leur domaine d'intervention. Voir également: Ollitrault, (S.), «Les écologistes français. Des experts en action », Revue française de science politique, 2001, 51(1-2), 2001, pp.105-131.
} 
déconcentrés en propre pour l'application et le contrôle de la législation ${ }^{3}$. Les lois de 1995 ont, par la suite, renforcé et étendu les modalités de participation des associations ${ }^{4}$.

On est loin des conceptions classiques de la souveraineté nationale, lesquelles excluaient que la volonté générale puisse être troublée par l'intervention de corps intermédiaires ${ }^{5}$. Rien ne devait en principe s'interposer entre l'État et les citoyens. En France, depuis la Révolution qui abolit les ordres et les corporations jusqu'en 1901, ceux-ci étaient en effet déconsidérés. Les sociétés ou les associations qui défendaient des intérêts concrets, particuliers, étaient même interdites car suspectées de nuire à l'expression de l'intérêt général. La loi de 1901 sur la liberté d'association, qui régit encore les groupements à but non lucratif, marque un grand changement. Peu à peu, les actions des associations, qu'elles soient sportives, culturelles, philanthropiques ou naturalistes, ont été reconnues d'intérêt commun. Dans certains domaines, ces dernières se sont affirmées comme des partenaires indispensables des autorités, faisant remonter des revendications susceptibles d'être inscrites à l'agenda, assurant parfois des missions de service public.

Les fédérations de pêche ont été parmi les premières à voir leurs activités ainsi valorisées. Créées en 1941 par la loi pêche, elles sont chargées de collecter la taxe piscicole dont une partie est destinée à l'entretien des milieux aquatiques. En 1964, le législateur, qui souhaitait élaborer une grande loi sur l'eau tenant compte des bassins hydrographiques, a été obligé d'impliquer les pêcheurs dans la gestion des ressources aquatiques ${ }^{6}$. De par leur loisir qui suppose une longue présence sur le terrain, une attention particulière aux peuplements piscicoles, ces derniers sont en effet les principaux témoins de l'évolution de l'état des cours d'eau. Ils sont directement concernés par leur qualité et constituent de fait des relais précieux des autorités sur le terrain. Par des pressions régulières ils ont pu faire valoir leurs compétences et s'imposer comme des interlocuteurs légitimes ${ }^{7}$. De la même façon, les membres des associations de protection de la nature favorisées par les lois de 1976 et 1977 sont des témoins privilégiés de l'état des milieux et des espèces qu'ils représentent. L'État les a également eu intérêt à les désigner comme ses partenaires.

Ce faisant, on peut dire que les législations sur l'eau et sur l'environnement ont considérablement bouleversé nos façons de penser puisqu'elles ont officiellement reconnu, dès la fin des années 1960, les pêcheurs et les environnementalistes comme les porte-parole d'entités non-humaines dans des domaines bien spécifiques. Il ne s'agit pas là de porte-parole universels dotés d'une compétence généralisée, mais de personnes qui, grâce à la pratique d'un loisir ou à leur engagement sont dépositaires de savoirs et de savoir-faire techniques particuliers, souvent développés dans la proximité, et qui vont pouvoir parler au nom d'entités muettes comme les poissons et les rivières ${ }^{8}$. Cette habilitation à «parler pour » s'est précisée

\footnotetext{
${ }^{3}$ Lascoumes, (P.), L'écopouvoir. Environnements et politiques, La Découverte, Paris, 2004.

${ }^{4}$ Notamment pour ce qui concerne la consultation en amont des projets d'aménagement. Voir Braud, (X.), « Les droits des associations : cadre renouvelé en 95. De la vigilance citoyenne en matière d'environnement », Le Louarn, (P.), (dir.), Décision locale et droit de l'environnement, Rennes, PUR, 1998.

${ }^{5}$ Barthélémy, (M.), Associations : un nouvel âge de la participation ?, Presses de Science-Po, Paris, 2000.

${ }^{6}$ La loi sur l'eau de 1964 n'a pas été votée ex nihilo mais hérite et prolonge, tout en les articulant, des principes épars de gestion, pour certains très anciens, comme ceux relevant de la pêche. Pour une analyse des lois sur l'eau s'étant succédées depuis 1964, voir Le Bourhis, (J.-P.), La publicisation des eaux. Rationalité et politique dans la gestion de l'eau en France (1964-2003), Thèse de science-politique, Université Panthéon-Sorbonne, Paris, 2004.

${ }^{7}$ Bouleau (G.), « Le rôle des pêcheurs aux origines de la loi sur l'eau de 1964 », Economie rurale, à paraître.

${ }^{8}$ Pour une réflexion sur les transformations de l'espace public et de l'engagement, voir Ion, (J.), La fin des militants ?, Paris, les Editions de l'atelier et les Editions ouvrières, Paris, 1997 ; Karpik, (L.), « Les nouveaux porte-parole », Fondation Saint-Simon, France : les révolutions invisibles, Paris, Calmann-Lévy, 1998. Pour ce qui concerne les savoirs des pêcheurs, on se réfèrera, à la suite des réflexions engagées par Laurent Thévenot sur le proche, aux travaux de Doidy, (E.), «La vulnérabilité du sujet politique. Régimes de proximité dans les arènes d'engagement public », Thèse de sociologie, EHESS, Paris, 2002; on pourra également voir ceux de
} 
avec les années, à mesure qu'émergeaient et étaient problématisées les questions environnementales. Si dans un premier temps, il s'agissait surtout pour les pêcheurs de veiller à la bonne santé des poissons, depuis, les pêcheurs, les naturalistes et les environnementalistes ont enrôlé un collectif élargi d'êtres - poissons, crustacés et végétaux - pour leur servir d'indicateurs de la qualité de l'eau des rivières.

Avec l'émergence des questions environnementales, le nombre des médiations, mais également des porte-parole, s'est accru. C'est notamment le cas dans des espaces autrefois dominés par l'agriculture. D'où le questionnement suivant : comment le «rural» qu'on a longtemps défini en négatif parce que moins peuplé et parce qu'il était l'image inversée des villes, se manifeste-t-il et qui peut le faire parler? Sont-ce les agriculteurs qui habitent majoritairement ces espaces longtemps dédiés à la production des animaux et des plantes ${ }^{9}$ ? Que faire alors des ingénieurs agricoles, des semenciers, des marchands de produits phytosanitaires, des firmes agroalimentaires, des nouveaux ruraux comme on les appelle, des environnementalistes, des promeneurs et même des sociologues? Tous concourent à «énacter» des versions différentes de la ruralité ${ }^{10}$. L'espace rural s'est diversifié. En même temps qu'il se peuplait de machines et de nouvelles entités hybrides de nature et de technologie, qu'il se transformait, de nouveaux conflits d'usage sont apparus et avec eux de nouvelles revendications. ${ }^{11}$.

Il ne sera pas ici seulement question des relations entre les pêcheurs ou entre des humains qui utilisent des milieux qu'on pourrait qualifier de ruraux. Nous évoquerons bien entendu les actions militantes des membres de l'Association nationale de protection des eaux et rivières (ANPER-TOS, nommée précédemment TOS pour Truites, ombres et saumons) mais nous tâcherons surtout d'examiner leurs effets sur ces mêmes milieux. Que savons-nous vraiment des environnements sur lesquels portent les procès ? Sont-ils de simples décors inertes? Qu'est-ce que les affaires comme celle de Chanac-concerne une porcherie industrielle- nous apprennent des non-humains qu'elles mobilisent ? Comment ces derniers transforment à leur tour les humains et nous invitent à repenser nos savoirs sur les mondes ruraux ? Nous décentrerons le regard pour prendre en compte non seulement les interactions entre les humains, mais également les interactions entre tous les autres êtres. Nous montrerons comment les agencements singuliers ainsi produits, hybrides d'humains et de non-humains, peuvent agir et constituer des versions différentes de ce que l'on qualifie de « rural » par commodité, faute de terme qui embrasse la multiplicité des êtres que cela concerne.

\section{Vers une redéfinition positive de la notion de porte-parole}

La notion de porte-parole, classique en science politique quand il s'agit de discuter de démocratie représentative, a fait l'objet de nouvelles analyses avec le développement de la sociologie des sciences $^{12}$. Dans cette perspective, le porte-parole n'est plus forcément

Barthélémy, (C.), Des rapports sociaux à la frontière des savoirs. Les pratiques populaires de pêche amateur au défi de la gestion environnementale du Rhône, Thèse de sociologie, Université de Provence, Marseille, 2003.

9 Mendras, (H.), La fin des paysans, Arles, Actes Sud, 1984 ; Jollivet, (M.), « La vocation actuelle de la sociologie rurale », Ruralia, n $6,1997,15 \mathrm{p}$.

${ }^{10}$ Nous empruntons ce terme à l'anglais dont le sens est proche mais plus fort que celui de performation pour désigner les processus et les pratiques qui concourent à la production des choses et des réalités qui les accompagnent. Voir Mol, (A.), The Body Multiple: Ontology in Medical Practice, Durham, Duke University Press, 2002.

11 Rautenberg, (M.), Micoud, (A.), Bérard, (L.), Marchenay, (P.) (dir.), Campagnes de tous nos désirs. Patrimoines et nouveaux usages sociaux, Paris, MSH, 2000.

${ }^{12}$ Pour une introduction, voir Latour, (B.), La science en action, Paris, Gallimard, 1995. Pour une présentation de cas issus de l'histoire des sciences, voir Daston, (L.) (ed.), Things That Talk. Lessons from Art and Science, New York, Zone Books, 2004. 
l'humain qui exprime les idées d'un autre humain ou d'un groupe humain, c'est autant le délégué des marins-pêcheurs de Saint-Brieuc que "quelques larves sur quelques collecteurs considérées comme les représentants crédibles d'une masse silencieuse et insaisissable de coquilles tapies au fond des eaux océanes ${ }^{13}$. La portée de la notion est donc toute autre. On trouve d'ailleurs de nombreux exemples dans la sociologie des sciences qui montrent des scientifiques occupés à produire des instruments pour faire parler les choses. Ainsi, pour Bruno Latour, «les blouses blanches ont inventé (et ne cessent d'inventer) des appareils de phonation qui permettent aux non-humains de participer aux discussions des humains ${ }^{14}$.

En matière d'environnement, on observe des phénomènes semblables. On ne peut pas se passer de porte-parole puisque la majorité des êtres concernés n'est pas directement visible. De fait, la sociologie si elle veut comprendre ce qu'il advient des environnements auxquels elle est confrontée, doit s'intéresser sérieusement aux médiations qui permettent à des êtres d'exprimer les préférences d'autres êtres. Par exemple, les écotoxicologues fabriquent des indicateurs pour mesurer la qualité de l'eau, ils enrôlent des organismes sentinelles pour les y aider ${ }^{15}$. La notion de «bon état écologique de la rivière » est produite grâce à la mise en rapport de différentes informations obtenues au cours d'un processus d'instrumentation et d'observation permettant à une masse d'eau de manifester ses propriétés, ses vulnérabilités et ses exigences ${ }^{16}$. Le problème, c'est que cette mobilisation en cascade est pensée en dehors de la politique. Alors que justement, quand les naturalistes et les pêcheurs parlent au nom de ceux auxquels ils sont attachés, ils formulent des propositions qui mettent à l'épreuve nos définitions du monde commun et, au-delà, le partage ancien entre nature et société ${ }^{17}$.

Comme avec n'importe quel porte-parole, il convient de douter de la capacité des porte-parole à parler au nom de tiers. Toutefois, contrairement à ce qu'en aurait dit Pierre Bourdieu, le porte-parole ne confisque pas forcément la parole à son profit. Il opère des traductions qu'il s'agit de repérer et de suivre. Plus ses efforts pour rendre les choses éloquentes auront été questionnés, testés et rectifiés collectivement, meilleur pourra ensuite être notre jugement sur elles. On pourra alors se demander comment cohabiter avec ces êtres nouveaux qu'il nous donne à entendre, décider des procédures à mettre en œuvre pour distinguer entre des traductions fiables, qui donnent à voir et penser leurs conditions de production, et d'autres qui sont moins robustes.

Notre approche s'inspire de la sociologie des sciences, mais également de tous les travaux qui traitent des objets ou des choses et qui, ce faisant, considèrent que l'on n'agit jamais seul, que l'action est forcément distribuée, comme l'agency ${ }^{18}$. Cela nous oblige avant toute chose à prendre au sérieux les acteurs, à ne pas leur imputer des intentions cachées. Cela

\footnotetext{
${ }^{13}$ Callon, (M.), «Eléments pour une sociologie de la traduction. La domestication des coquilles Saint-Jacques et des marins-pêcheurs de la baie de Saint-Brieuc », L’Année sociologique, 1986, n³6, pp.169-208.

${ }^{14}$ Latour, (B.), Politiques de la nature, Paris, La Découverte, 1999.

${ }^{15}$ Gramaglia, (C.), «River Sentinels: Finding a Mouth for the Lot River », Latour, (B.), Weibel, (P.) (eds.), Making Things Public. Atmospheres of Democracy, Cambridge, ZKM, MIT Press, 2005.

${ }^{16}$ Nous avons choisi la traduction du terme anglais «water body » pour embrasser toute la diversité des êtres qu'ANPER-TOS entend défendre sous toutes leurs formes. Nous verrons qu'un des effets de l'action de l'association est de qualifier des êtres qui ne l'étaient pas, de leur attribuer des propriétés et de faire respecter la règlementation qui les concerne. On retiendra que le terme est aujourd'hui utilisé dans la Directive cadre européenne sur l'eau (DCE) dans un souci de protéger non seulement les rivières mais aussi leurs affluents et leurs exutoires, soit solidairement l'ensemble du réseau hydrographique.

${ }^{17}$ Latour, (B.), «De la politique du temps à la politique de l'espace ? », Rémy, E., November, V., D’Alessandro, C., Charvolin, (F.), Espaces, savoirs et incertitudes, Paris, Ibis Press, 2005.

${ }^{18}$ On se réfèrera ici à l'ouvrage collectif dirigé par Conein, (B.), Dodier, (N.) et Thévenot, (L.) (dir.), Les objets dans l'action. De la maison au laboratoire, Paris, EHESS, 1993. On pourra également aller voir Barbier, (R.), Trépos, (J.-Y.), «Humains et non-humains: un bilan d'étape de la sociologie des collectifs », Revue d'anthropologie des connaissances, ${ }^{\circ} 1,2007$, pp.11-34.
} 
nous engage aussi à produire des descriptions fines à partir des matériaux empiriques récoltés, comme nous le ferons pour l'affaire de Chanac.

Pour notre recherche, nous avons régulièrement fréquenté pendant deux ans le local de l'association ANPER-TOS à Alforville de façon à observer le travail quotidien de son délégué. Nous avons assisté aux réunions de l'association. Nous avons également réalisé quarante-sept entretiens semi-directifs avec des membres d'ANPER-TOS et des personnes impliquées dans certaines des affaires qu'elle avait contribué à lancer. Nous avons ensuite dépouillé les archives de six procès ce qui nous a permis de suivre les échanges entre les parties prenantes. Nous nous sommes intéressée aussi bien à la passion pour la pêche à la mouche, aux connaissances qu'elle génère qu'aux actions contentieuses, aux effets qu'elles ont produits sur les environnements qu'elles visaient. Nous avons bien entendu étudié le fonctionnement d'ANPER-TOS mais nous n'aurons pas le temps d'en parler ici. Plutôt que de comparer cette association à d'autres selon des critères organisationnels, nous avons préféré comprendre en quoi ce qu'elle fait transforme les environnements dont elle se saisit et le droit lui-même qui lui sert à la fois d'instrument et de tactique ${ }^{19}$. Nous voudrions ici défendre une sociologie qui ne considèrerait pas les environnements comme un objet déjà assemblé et stable, mais une sociologie qui s'intéresserait à la production de différents environnements et de la ruralité dans leurs dimensions inextricablement humaines et non-humaines. La politisation dont nous entendons traiter n'est pas seulement le fait de l'arrivée de nouveaux acteurs humains dans les campagnes, mais plutôt la conséquence d'une irruption, celle du monde matériel qui commence à poser problème, qui résiste aux usages que l'on veut en faire et qui exprime, indirectement, ses vulnérabilités et ses exigences.

Cet article se propose donc d'aller voir comment, à l'occasion d'une pollution affectant une nappe phréatique, les propriétés de l'eau en tant qu'être et en tant que milieu ont pu être explicitées. Nous concentrerons notre attention sur ANPER-TOS qui a agit, à cette occasion, comme porte-parole. Nous commencerons par retracer l'histoire de ce groupement de pêcheurs à la mouche et de juristes, puis nous détaillerons les moyens d'action dont il dispose. L'examen d'un procès qui l'a mobilisé aux côtés d'une autre association de riverains, l'affaire de Chanac qui s'est déroulée en Lozère, nous permettra de montrer comment, sous certaines conditions, les milieux ruraux et les êtres qui les constituent peuvent devenir éloquents, faire leur entrée en politique. Nous insisterons particulièrement sur le travail de composition accompli par ANPER-TOS qui, grâce à l'usage tactique du droit, est parvenue à pointer des interdépendances entre les humains et les non-humains, et à rouvrir la question du monde commun.

\section{Les transformations d'une association fondée par des pêcheurs passionnés}

ANPER-TOS est à la fois unique de par son histoire et emblématique d'une grande partie des associations dites de protection de la nature, tout au moins de celles dont le travail principal consiste à produire des revendications. Créée en 1958 par des notables passionnés de pêche à la mouche, ANPER-TOS s'appelait à l'origine TOS, en référence aux salmonidés, poissons qui avaient les faveurs de ses membres ${ }^{20}$. Ses activités n'étaient pas très différentes de celles des autres groupements de pêcheurs de l'époque. On y échangeait des informations et des idées de parcours de pêche à la mouche. Rapidement, ANPER-TOS s'est pourtant distinguée par ses prises de position critiques. En même temps qu'elle faisait la promotion de

\footnotetext{
${ }^{19}$ Par tactique nous désignons des usages rusés des procédures qui permettent de renverser un rapport de force défavorable, d'obtenir des informations ou d'autres ressources, et même de faire jouer le droit contre le droit pour l'amender et le faire progresser.

${ }^{20}$ Les salmonidés, truites, ombres, et saumons, ont pour autres caractéristiques de vivre dans des eaux froides de très bonne qualité. Ils deviennent, de par leur seule présence, des indicateurs de l'état de santé des cours d'eau.
} 
la pratique de la pêche à la mouche, elle défendait des valeurs sportives centrées sur la modération et l'attention portée aux espèces piscicoles. Les membres fondateurs de cette association étaient pour beaucoup des notables. Ils aimaient se retrouver entre pairs. Ils s'étaient regroupés pour inciter leurs homologues pêcheurs à respecter la règlementation, dans un esprit de fair-play mais également pour préserver les ressources halieutiques. Ils les ont également encouragés à contempler les paysages pittoresques que leur pratique les amenait à fréquenter. Ce faisant, ils ont contribué à transformer les rapports que les pêcheurs entretenaient avec les rivières et les espaces où coulent celles-ci ${ }^{21}$.

ANPER-TOS s'est rendue visible en se dotant d'un organe de liaison, publié au sein de la revue Plaisirs de la pêche. Ainsi, elle a pu diffuser ses idées. Ce qui n'était au début que quelques feuillets, a progressivement gagné en importance et a servi de lieu de rencontres et de débats. Les grandes rivières françaises et étrangères ont été mises à l'honneur dans des articles bien documentés, en fonction des voyages entrepris par ses membres. Toutefois, à partir des années 1960 et plus encore 1970, l'association a été amenée à donner un tour plus protestataire à ses actions. Elle a multiplié les démarches pour obtenir une réglementation plus restrictive en matière de protection des rivières, notamment les rivières de première catégorie, dites salmonicoles ${ }^{22}$. Elle a œuvré pour l'interdiction de la commercialisation de la truite sauvage et le relèvement de la taille des prises pour les salmonidés, usant pour cela des relations de certains de ses membres avec des élus. Elle a ensuite pris position contre le développement des microcentrales hydrauliques.

Nous n'insisterons pas sur les origines socio-professionnelles des membres de l'association. Il suffit pour notre propos de dire que certains de ses fondateurs étaient des notables, c'est-à-dire des chefs d'entreprise et des hauts fonctionnaires qui bénéficiaient de relations privilégiées avec des élus. C'est ce qui explique que les autorités aient accédé rapidement à certaines de leurs demandes. Par la suite, ANPER-TOS a beaucoup changé. Son mode de recrutement s'est assoupli en même temps qu'elle explorait d'autres modes d'action, qu'elle se faisait plus revendicative. Dès lors, l'association n'a cessé d'évoluer. Grâce aux contributions critiques publiées dans la revue spécialisée qu'elle a créée, TOS, et aux nombreux courriers qu'elle a adressés aux autorités, elle s'est faite un nom, à la fois dans les milieux de la pêche et parmi un groupement émergeant, celui des protecteurs de la nature. ANPER-TOS est aujourd'hui une association composite qui rassemble environ sept-cent membres. Ce sont pour la plupart des pêcheurs qui pratiquent la mouche et se contentent de payer leur cotisation. À l'occasion, ils font remonter des informations sur l'état des rivières. Ils veillent et réunissent des preuves qui servent dans la constitution des dossiers et des affaires. On trouve parmi eux un bon tiers de cadres ou professions intellectuelles supérieures comme dans beaucoup d'associations de protection de la nature, mais toutes les catégories socio-professionnelles sont représentées. On doit cependant noter que parmi les personnes qui ont le plus influencé l'évolution d'ANPER-TOS, se trouvent plusieurs autodidactes, dont un pêcheur à la mouche passionné qui, le premier, s'est intéressé aux possibilités offertes par le droit. C'est lui qui a engagé le premier procès à l'encontre des industriels responsables de la dégradation de la qualité de sa rivière préférée l'Ellé, en Bretagne. C'est également lui qui a insisté pour développer les actions contentieuses. Il a créé une commission Pollution et nuisance au sein d'ANPER-TOS qui s'est spécialisée dans la préparation des procès. Depuis,

\footnotetext{
${ }^{21}$ On trouve de nombreux exemples dans la littérature qui décrivent l'influence des pratiques de loisir sur le changement des savoirs et pratiques en lien avec la «nature. On se réfèrera à Thomas, (K.), Dans le jardin de la nature. La mutation des sensibilités en Angleterre à l'époque moderne (1500-1800), Paris, Gallimard, 1985 et à Corbin, (A.), L'avènement des loisirs. 1850-1960, Paris, Flammarion, 1995.

${ }^{22} \mathrm{~L}$ 'administration attribue aux rivières une note relative à la qualité de l'eau et à la diversité des peuplements (1A: excellente, 1B: bonne, 2: passable / reproduction piscicole limitée, et 3: médiocre / vie piscicole menacée). Il existe une catégorie dite « hors classe » pour celles qui sont très dégradées et présentent un risque.
} 
de nombreux juristes ont adhéré à l'association après avoir été invités à participer à un colloque organisé conjointement avec le Syndicat de la magistrature, en 1988. Ces professionnels du droit, bien que peu intéressés par la pêche, partagent avec les pêcheurs une indignation commune, celle générée par les pollutions, elles-mêmes souvent causées par des infractions au droit de l'environnement.

En effet, à mesure que l'industrialisation progressait, la qualité de l'eau s'est dégradée. Les Trente Glorieuses, considérées comme une deuxième grande phase d'industrialisation, ont en effet cruellement mis à mal les rivières françaises ${ }^{23}$. Les cas de pollution se sont multipliés et les pressions sur les milieux se sont accrues. Les peuplements piscicoles ont diminué. Indignés, littéralement empêchés de se livrer à leur loisir préféré - le terme revient suffisamment souvent dans les entretiens pour le noter - les pêcheurs de l'association ANPER-TOS ont cherché des moyens plus efficaces d'agir. Ils ne sont cependant pas passés d'un mode d'action à un autre par une prise de conscience purement intellectuelle qui leur aurait permis de monter en généralité ${ }^{24}$. Il a fallu qu'ils fassent l'expérience de la pollution, qu'ils soient directement touchés, qu'ils se laissent eux-mêmes affecter par les dégradations menaçant les salmonidés et leur milieu. Alors, ils ont pu envisager d'agir en leur faveur. De porte-parole des poissons qu'ils affectionnaient, ils sont devenus les porte-parole de toutes les espèces piscicoles et des masses d'eau en tant qu'assemblages d'êtres hétérogènes.

Après avoir multiplié les démarches et les pressions ciblées, ils ont traduit leurs revendications en plaintes qu'ils sont allés porter devant la justice. Certains pêcheurs ont même arrêté de pêcher, consacrant tout leur temps à l'apprentissage des textes de lois et des procédures. C'est pour saisir toutes ces transformations, et les inévitables tensions qu'elles provoquent -entre pêcheurs qui veulent conserver les caractéristiques anciennes du club halieutique et ceux qui se passionnent pour le doit, qui sollicitent des juristes- que nous nous sommes intéressée aux trajectoires des personnes concernées et aux processus plus qu'aux catégories toutes faites. Nous avons ainsi pu voir comment des acteurs individuels s'associent et forment un collectif plus puissant, une association de protection de la nature qui peut, sous certaines conditions et malgré la diversité des feuilletages qui la composent, "parler et agir comme un seul homme ${ }^{25}$.

\section{L'agrément en tant qu'habilitation à «parler pour » et instrument militant}

L'évolution de la législation a donné aux associations de protection de la nature la possibilité d'engager des poursuites à l'encontre de tout contrevenant qui porterait atteinte aux intérêts qu'elles représentent. Outre la reconnaissance d'utilité publique, procédure sélective permettant de recevoir des subventions, des dons ou des legs, l'agrément constitue un outil supplémentaire, autorisant celles qui en bénéficient à participer à la gestion d'organismes publics mais également à intenter des actions en justice ${ }^{26}$. Elles peuvent ainsi, du moins en principe, agir comme de véritables « auxiliaires de justice »en complément des polices administratives chargées de veiller au respect de la loi en matière d'environnement. L'agrément est une fiction juridique qui fait des associations les porte-parole d'entités nonhumains qui autrement seraient exclues de la représentation politique.

\footnotetext{
${ }^{23}$ Boullet, (D.), Entreprises et environnement en France de 1960 à 1990. Les chemins d'une prise de conscience, Thèse d'histoire contemporaine, Université Paris X, Nanterre, 1999.

${ }^{24}$ Thévenot, (L.), « Mettre en valeur la nature. Disputes autour d'aménagements de la nature en France et aux Etats-Unis », Autre temps, n49, 1996, pp.27-50.

${ }^{25}$ Callon, (M.), Latour, (B.), «Le grand Léviathan s'apprivoise-t-il ? », Sociologie de la traduction. Textes fondateurs, Akrich, (M.), Callon, (M.), Latour, (B.), Paris, Presses de l'Ecole des Mines, pp.11-32.

26 Léost, (R.), «L'agrément des associations de protection de l'environnement», Revue juridique de l'environnement, $\mathrm{n}^{\circ} 2,1995$, pp.265-290.
} 
L'agrément se définit à la fois comme habilitation et comme «présomption légale d'intérêt à agir ${ }^{27}$. Grâce à l'abondance relative des textes juridiques ayant trait à l'environnement, il offre aux associations de protection de la nature, des moyens d'action inédits et des voies de recours additionnelles ${ }^{28}$. Le droit se présente en effet à la fois comme un cadre à partir duquel les associations comme ANPER-TOS peuvent formuler leurs griefs et leurs plaintes, mais il leur offre également une arène où les exprimer. L'action contentieuse permet alors à celles qui le veulent de convoquer leurs adversaires devant un juge ou tiers arbitre, mais également d'interpeller les pouvoirs publics, soit directement en les assignant devant le tribunal administratif, soit indirectement en rendant publiques des informations qui ne l'étaient pas et qui vont, du fait même de leur publicisation, contraindre ces mêmes pouvoirs publics à réagir.

ANPER-TOS a bénéficié de l'agrément dès 1978. Elle n'a pas attendu pour s'en servir. Elle l'a fait dès qu'elle a pu obtenir, grâce à la Commission d'accès aux documents administratifs (CADA) les pièces nécessaires produites par les services de l'État, soit principalement les préfectures, les DRIRE et les DDAF ${ }^{29}$. Celles-ci lui ont servi de base, pour contester des arrêtés qu'elle trouvait illégitimes, pour vérifier les prescriptions applicables aux installations classées ainsi que des mesures et des analyses. Car, l'association n'a pas les moyens de commander elle-même des études. Elle se contente de rassembler et de connecter des informations produites par d'autres, comme des rapports demandés par l'administration, de manière à faire sortir des dossiers restés en sommeil.

Le nombre des procès engagés par ANPER-TOS depuis la fin des années 1970 dépasse la centaine. Ils peuvent être qualifiés d'affaires, au sens donné à ce terme par Elisabeth Claverie, c'est-à-dire de "de chose jugée au tribunal, puis contestée de l'extérieur ${ }^{30}$. C'est que les affaires se constituent à la fois dans le droit et en dehors de lui. En tant que tel, le droit ne peut rien. Il doit être activé. Il ne devient effectif qu'une fois lesté de nombreux d'adjuvants ${ }^{31}$. Pour ANPER-TOS, chaque action en justice commence par une enquête de terrain destinée à collecter des éléments qui, une fois mis en rapport les uns avec les autres, requalifiés et enchâssés dans un récit vont acquérir une valeur juridique, former des preuves. L'association fait également au préalable un travail d'intéressement en direction des parties prenantes et des populations locales. Elle cherche des soutiens qui viendront nourrir ses plaintes de nouveaux éléments et incarner sur le terrain les intérêts qu'elle défend, c'est-àdire les êtres dont elle s'est faite le porte-parole.

\footnotetext{
${ }^{27}$ L'agrément et la reconnaissance d'utilité publique peuvent être retirés aux associations qui ne se montreraient pas dignes d'en bénéficier, soit qu'elles en font un usage jugé abusif, soit que leur action ne correspond pas aux missions qu'elles ont annoncées. Pour certains, comme les autres formes de participation institutionnalisée, l'agrément contribue à déconstruire les groupes d'opposants en une série d'acteurs individualisés. Il favorise les plus modérés et tend à euphémiser les conflits. Pour une analyse critique tirée des mobilisations antinucléaires des années 1970, voir Pollak, (M.), Nelkin, (D.), The Atom Besieged. Extraparliamentary Dissent in France and Germany, Cambridge, MIT Press, 1984.

${ }^{28}$ Voir Spanou, (C.), « Le droit instrument de la contestation sociale ? Les nouveaux mouvements sociaux face au droit », CURAPP, Les usages sociaux du droit, Paris, PUF, 1989 ; Coglianese, C., « Social Movements, Law and Society : the Institutionalisation of the Environmental Movement », University of Pennsylvania Law Review, $\mathrm{n}^{\circ} 150,2001, \mathrm{pp} .85-118$.

${ }^{29}$ Ces sigles désignent la Direction régionale de l'industrie, de la recherche et de l'environnement, et la Direction départementales de l'agriculture et des forêts.

${ }^{30}$ Voir Claverie, (E.), « Procès, affaire, cause. Voltaire et l'innovation critique », Politix, n²6, 1994 ; pp.76-85 ; Gaïti, (B.), Israël, (L.), « Sur l’engagement du droit dans la construction des causes », Politix, n 16(62), 2003, pp.17-30.

31 Pour une présentation du droit comme système sémiotique, voir Landowski, (E.), La société réfléchie, Paris, Seuil, 1988 ; Pour une réflexion sur les compétences du droit et sur les conditions de sa réalisation à partir de l'exemple du contentieux associatif en matière d'environnement, voir Gramaglia, (C.), La mise en cause environnementale. Casuistique des affaires de pollution de rivières : l'exemple des actions contentieuses de l'ANPER-TOS, Thèse de sociologie, Ecole des Mines, Paris, 2006.
} 
ANPER-TOS est à la fois une association nationale dont le siège est localisé en région parisienne, dont le conseil d'administration est aujourd'hui en majorité dirigé par des juristes professionnels, et un réseau de pêcheurs-veilleurs présents sur le terrain. Le délégué présent à Paris assure l'articulation des attentes et des besoins du groupement. Sa mobilité, le fait qu'elle ne soit pas gênée par des accointances avec des acteurs locaux comme les fédérations de pêche, la rend plus efficace. Elle est suffisamment présente localement pour préparer au mieux ses plaintes, mais elle est aussi mobile, détachée des obligations qui parfois s'imposent dans la proximité. Après quelques années d'hésitation et d'apprentissage, ANPER-TOS s'est donc spécialisée dans les recours et les coups juridiques. Elle a également changé ses statuts en 1980 et 1999 pour devenir une association de protection de la nature à part entière. Cela lui a notamment permis d'élargir son champ d'action à la défense des milieux aquatiques et de l'eau sous toutes ses formes. Elle se présente désormais comme une association de spécialistes à la fois reconnus et craints pour leur habileté juridique, leur opiniâtreté. Si elle ne remporte pas tous les procès qu'elle engage, du moins parvient-elle à faire entendre jusque dans les prétoires la parole des poissons, des rivières et des nappes. Ses interventions publiques ont un caractère militant indéniable même si elles sortent des cadres ordinaires de la mobilisation $^{32}$. L'accumulation des procès et des affaires l'aide à familiariser les magistrats avec des questions environnementales souvent très techniques. Elle permet également que des données scientifiques et techniques relatives à la bonne santé des milieux aquatiques pénètrent le droit. Elle contribue surtout à rendre effectif le droit de l'environnement qui, beaucoup de commentateurs autorisés s'en émeuvent, est encore peu ou mal appliqué ${ }^{33}$.

\section{Comment émerge et se construit une affaire}

L'affaire de la porcherie de Chanac s'est déroulée il y a peu, mais les faits consignés dans le dossier juridique font remonter les problèmes à son installation en 1974. Avant que l'élevage porcin ne s'industrialise, régulièrement du lisier s'écoulait sur la route à l'entrée du Causse de Sauveterre au lieu-dit la Nojarède. En 1996, une coopérative aveyronnaise fait l'acquisition des terrains et des bâtiments pour y installer plusieurs centaines d'animaux. Elle a pour cela engagé des travaux, sans toutefois solliciter l'autorisation du préfet, comme la loi sur les installations classées l'impose au-delà d'un certain seuil. Pour l'administration, il n'y avait rien à redire puisqu'une fosse à lisier allait être construite et améliorer la situation. Une simple déclaration semblait suffire.

Pour ANPER-TOS, dont un ancien président (également pêcheur et membre de la Loutre chanacoise, une association de pêche locale) s'était établi dans le village une fois la retraite venue, il y avait cependant de quoi s'inquiéter. Il s'agissait en effet de la première porcherie industrielle implantée en Lozère. L'association avait en tête d'autres affaires survenues en Bretagne. Elle craignait que les problèmes de pollution liés aux élevages

\footnotetext{
${ }^{32}$ Le droit est de plus en plus mobilisé à des fins militantes. Les mouvements des « sans » et les opposants aux OGM, par exemple, ont eu recours au droit pour des raisons tactiques, pour faire jouer le droit contre le droit et renverser un rapport de force qui leur était défavorable (voir Mathieu, (L.), Comment lutter ? Sociologie et mouvements sociaux, Paris, Textuel, 2004). ANPER-TOS fait la même chose. Cependant, l'association se distingue en cela qu'elle a fait du droit son instrument, sa tactique mais aussi l'objet principal de ses actions. Elle travaille à le rendre plus effectif, à l'étendre et à le préciser.

${ }^{33}$ Pour une perspective d'ensemble sur les problèmes que pose la saisie des questions d'environnement par le droit, voir Ost, (F.), La nature hors la loi. L'écologie à l'épreuve du droit, Paris, La Découverte, 2003. Pour des commentaires de praticiens, voir Matharan, (X.), « La justice pénale et la police de l'environnement : le constat d'un divorce, les prémisses d'une union », Les cahiers de la sécurité intérieure, n9, 1992, pp.71-84; Busson, (B.), «Le mauvais procès des recours des associations : faux arguments et vraies menaces », Revue juridique de l'environnement, $\mathrm{n}^{\circ} 1,2001$, pp.59-71; Jegouzo, (Y.), «Le juge administratif et l'ordonnancement du droit de l'environnement », Revue juridique de l'environnement, n spécial, 2004, pp.19-30.
} 
intensifs ne se reproduisent dans ce département, relativement peu peuplé et considéré comme un «pays de sources » ${ }^{34}$. Localement, les réactions étaient pourtant faibles. La Lozère est un département qui peine à se développer économiquement. Les agriculteurs locaux soutenaient le projet, pensant que cela ouvrait de nouvelles perspectives pour eux. L'extrait d'entretien présenté ci-dessous témoigne des tensions qui ont surgi lorsque l'un des leurs, qui avaient déjà pris ses distances en optant pour l'agriculture biologique, s'est joint aux opposants.

«Les autres, ils se sont dit: 'on a de la chance que des gens aient accepté de venir investir pour développer une activité chez nous, dans notre coin, alors qu'on n'a pas été capable de le faire...Et puis, c'est un bel outil, ça produit, ça créé des emplois et on peut même enrichir nos terres'. Aussi, ça les a choqué qu'un agriculteur comme eux prenne position contre la porcherie. Quand les voisins ont vu ça dans la presse locale, et bien croyez-moi que ça m'a valu des réprimandes et des méchancetés » (ROBERT, ANPI, Association créée au moment de l'émergence de l'affaire).

Après avoir mené l'enquête et essayé de problématiser l'affaire, c'est-à-dire identifier les enjeux et les traduire pour leur donner un sens qui interpelle le plus grand nombre, ANPER-TOS a décidé de déposer une requête devant le tribunal administratif de Montpellier. L'association a acquis au fil du temps une réelle connaissance des lois sur les installations classées auxquelles les élevages industriels de taille importante sont soumis. Elle s'est donc rapidement aperçue que le préfet n'aurait pas dû accepter que des travaux de construction et de rénovation soient réalisés sans au préalable diligenter une étude scientifique et déclencher une enquête publique. Après avoir saisi la CADA pour obtenir des documents, elle a formé, en 1997, un premier recours en annulation devant le tribunal administratif. L'année suivante, elle a également déposé une requête à l'encontre du maire du village pour contester la validité du permis de construire qui n'avait pas fait l'objet d'un affichage réglementaire indiquant à d'éventuelles parties lésées les délais pour se faire connaître ${ }^{35}$.

ANPER-TOS a dans un premier temps agi seule. Tout son travail a été de montrer, dans les différents mémoires échangés avec les services de la préfecture et la coopérative porchère, que l'autorisation octroyée n'était pas valable à cause des nombreuses erreurs de procédures qui l'entachaient. Aucune précision technique n'avait en effet été fournie concernant le fonctionnement de l'élevage et les risques environnementaux liés à l'épandage ${ }^{36}$. En l'occurrence, la transformation des places de porcs charcutiers en places de truies constituait à elle seule un motif d'annulation puisqu'il est connu que les animaux reproducteurs produisent plus de lisier que les autres. En réponse à ces accusations, l'administration a surtout cherché à mettre en doute la légalité et la légitimité de la requête d'ANPER-TOS, mentionnant d'abord les délais de recours dépassés puis son statut d'organisation nationale dénuée d'implantation locale.

En 1999, le juge a cependant révoqué la décision du préfet, considérant que les transformations faites par les nouveaux exploitants rendaient nécessaire l'instruction d'un

\footnotetext{
${ }^{34}$ La Lozère compte plus de deux mille kilomètres de rivières et de ruisseaux. Plusieurs grands cours d'eau français comme le Lot y naissent. La densité et la variété du réseau hydrographique, la faible pression industrielle qui s'y exerce, en font un département à part, un enjeu important pour l'association ANPER-TOS.

${ }^{35}$ Un même cas peut donner lieu à plusieurs procès qui se nourrissent l'un l'autre. ANPER-TOS joue consécutivement sur le tableau du droit et sur celui de la tactique. Ses membres préparent soigneusement leurs plaintes même s'ils sont contraints de bricoler faute de moyens matériels. Ils choisissent les juridictions et les moyens juridiques qui leur semblent appropriés. Ils rusent avec les procédures pour gagner du temps, obtenir des documents et des preuves supplémentaires.

${ }^{36}$ La loi impose que tout projet de développement d'un élevage industriel porcin soit accompagné d'un plan d'épandage pour l'élimination des déchets ainsi produits.
} 
nouveau dossier d'autorisation. L'annulation de l'arrêté préfectoral marque le début d'une bataille juridique qui a duré neuf ans en tout, puisque les porchers ont fait appel.

À l'issue du premier procès, une étude hydrogéologique a été commandée par le préfet au Bureau de Recherches Géologiques et Minières (BRGM) qui a souligné le caractère extrêmement fragile du sol et du sous-sol du Causse de Sauveterre. Une enquête publique a également été ouverte de décembre 1999 à janvier 2000, à Chanac et dans la commune voisine de Sainte-Enimie. ANPER-TOS, les associations de pêche locales et des spéléologues amateurs ont déposé lors de cette consultation, de même que quelques riverains isolés. En tout, les commissaires enquêteurs ont entendu seize personnes et reçu une quarantaine de lettres exprimant des inquiétudes. Ils ont rendu un avis favorable mais réservé. Trois personnes qui étaient venues déposer à titre individuel se sont cependant rencontrées à cette occasion. Elles ont décidé de fonder l'association Non à la Porcherie Industrielle de Chanacla-Nojarède $(\mathrm{ANPI})^{37}$. Elles ont également adhéré à ANPER-TOS pour bénéficier de ses compétences en matière juridique. Les deux associations se sont alors partagé les tâches. Chacune en fonction de ses possibilités a alimenté le dossier. L'objectif commun des deux associations était d'obtenir l'interdiction pour les porchers d'étendre leur exploitation, et surtout, d'épandre du lisier sur le causse de Sauveterre dont elles doutaient que le sous-sol puisse retenir durablement les polluants.

\section{Les usages militants du droit à l'intérieur et en dehors des tribunaux}

Les instruments que fournit le droit, ainsi que les usages tactiques qu'il autorise, présentent d'énormes avantages. Ils règlent notamment le problème de la représentation des non-humains, en désignant les associations de protection de la nature agréées comme leurs émissaires légaux. Par une fiction juridique, la reconnaissance des préjudices matériels et moraux de ces dernières, il reconnaît la valeur intrinsèque des liens qui associent des humains à des non-humains, et collectivise la qualification de victime qui sans ça, serait difficilement applicable (les atteintes aux milieux aquatiques et aux poissons ainsi que les troubles de jouissance des pêcheurs ne sont pas dissociés par le droit qui progresse en s'étendant de proche en proche, en fonction de différents régimes qui parfois se chevauchent ${ }^{38}$ ). Le droit permet en outre d'explorer, au cours de processus d'enquête collectifs, l'ensemble des causes et des responsabilités directes ou indirectes, à la source des problèmes dont il a à traiter. Dans le cas des problèmes environnementaux qui ont pour particularité d'allonger indéfiniment la chaîne des conséquences et des incertitudes, ce tâtonnement est absolument nécessaire. Celuici ne peut se faire qu'en intégrant un nombre très important d'acteurs, au-delà des seuls voisins concernés, cercle restreint d'ordinaire ciblé par les procédures d'enquête publique. Chaque problème environnemental dessine ses propres géographies, et ce faisant ses propres publics, au-delà des découpages administratifs existants. Il convient donc d'inventer des procédures $a d$ hoc permettant de les convoquer. Le tribunal est un des espaces où cela peut se faire. Ainsi, à Chanac, l'enquête publique n'avait pas permis l'agrégation des inquiétudes individuelles et l'organisation d'un débat contradictoire. C'est à son issue seulement que se sont regroupés les opposants. Ce n'est qu'ensuite qu'ils ont pu, collectivement, investiguer, mobiliser des ressources et rechercher des alliés. Le recours au contentieux a donc permis de

\footnotetext{
${ }^{37}$ C'est exemple montre que pour les riverains ou le public non organisé, ce type de consultation est inadapté. Il ne permet pas à des profanes de se saisir des dossiers à temps. Ainsi, dans beaucoup de cas, ANPER-TOS est intervenue après l'enquête. C'est dans les tribunaux qu'elle a obtenu leur réouverture.

${ }^{38}$ On se réfèrera au travail de Bruno Latour sur le sujet, La fabrique du droit, une ethnographie du Conseil d'Etat, La Découverte, Paris 2002 ; et au numéro spécial de la revue Cosmopolitiques sur le droit, n, 2004. Pour comprendre comment le droit se saisit des animaux selon différents régimes juridiques, voir Desmoulin, (S.), «Protection des animaux et condition juridique de l'animal en droit français », Revue interdisciplinaire d'études juridiques, n57, 2006, pp. 37-75.
} 
rouvrir une question préalablement réglée pour les autorités. Les tactiques que le droit autorise, plutôt que de renforcer les prérogatives des acteurs institués, ce qu'aurait tendance à dénoncer la sociologie critique, a donc ouvert des marges de manœuvres supplétives pour des acteurs minoritaires. Elles ont même permis que «s'expriment» par la bouche des porteparole, des êtres jusque-là exclus du débat.

«La plainte d'ANPER-TOS doit remonter à 1996...Le jugement est tombé deux ou trois ans plus tard. Le tribunal administratif a demandé au préfet de faire la régularisation au regard de la réglementation, et aux porchers de monter un dossier avec étude d'impact et de le soumettre à enquête publique. Celle-ci est fixée au 6 janvier 2001. C'est là que notre combat commence. Juste avant l'enquête publique, les Verts diffusent des tracts dans les boîtes aux lettres. Bien sûr, ils argumentent sur les dangers. J'ai découvert plein de choses...Notre association a été créée après... À la sortie de l'enquête publique. On s'est retrouvé à deux ou trois, et ça a commencé...» (BERNARD, ANPI).

ANPI a joué un rôle très important en maintenant la pression sur les élus locaux et en mettant à profit les compétences techniques de l'un de ses membres, également inspecteur des services vétérinaires. Celui-ci a analysé et critiqué en détail l'étude d'impact. ANPI a par ailleurs cherché à mobiliser les populations locales. Elle a sollicité le soutien des Verts de la Lozère et celui des touristes de passage, dont certains, d'origine bretonne, ont témoigné des problèmes de pollution agricole auxquels ils étaient confrontés dans leur département. Les deux extraits ci-après montrent deux moments de l'organisation, l'enquête sur les propriétés des êtres concernés et le déconfinement de la plainte.

«C'est les travaux qui m'ont alerté et surtout la construction d'une grosse fosse. Je me suis dit là il y a quelque chose...Le temps de se mettre en route et de récupérer l'arrêté d'autorisation à la préfecture. Réflexe de professionnel. Je voulais juste me tenir informé quand j'ai su que c'était un TA (tribunal administratif), et que c'était cassé. TOS était déjà intervenue...Mon expérience m'a permis de relever des points importants, sur la forme et le fond du dossier... À partir de ça on a pu fédérer quelques personnes. On a senti qu'il y avait la possibilité de faire fléchir le préfet... »(HERVE, ANPI).

«Pendant un an et demi, on fait signer des pétitions. On a fait les marchés de Chanac tous les dimanches de juillet et août 2000. On a fait un marché de nuit à Sainte-Enimie, la perle des gorges du Tarn. Le 20 décembre 2000, on avait trois mille quatorze signatures, dont mille cinq cents en Lozère. Tous les Bretons qui passaient, ils avaient compris. Ils disaient: on sort de Bretagne pour rechercher la nature, et on va retrouver ici des porcheries industrielles, il faut arrêter le massacre! »(BERNARD, ANPI).

C'est que les affaires comme celle de Chanac se nourrissent d'éléments extérieurs à partir desquels les textes juridiques sont mis à l'épreuve. ANPER-TOS a besoin de relais sur le terrain et même d'alliés pour lui fournir les renseignements lui permettant d'ajuster ses tactiques aux différents cas. Les opposants locaux lui apportent notamment des indications précises sur l'histoire des pollutions ou du projet contesté, et sur les spécificités environnementales des lieux. Ils lui transmettent également des informations sur la politique régionale, sur les élus ou sur les connivences éventuelles entre les autorités et d'autres parties prenantes. Ces alliés disposent en outre de compétences particulières que l'association met à profit. Car, le droit ne devient plus performant que si la plainte s'accompagne d'un travail de mobilisation à l'extérieur de l'arène juridique pour donner du poids et de la résonnance aux argumentations développées. Il est un moyen de construire un rapport de force différent de 
celui qui préexiste. Il ne remplace pas les moyens de lutte ordinaires. Il s'y rajoute. Les procès ne se gagnent donc pas seulement sur des points de droit, mais grâce au surcroît de crédibilité, à la force de conviction dont certaines argumentations vont être lestées par la mise en scène, les efforts de dramatisation supplémentaires qui vont permettre au juge de prendre la mesure des enjeux, d'évaluer l'opportunité de sa décision, puis de la motiver.

Les associations ANPER-TOS et ANPI ont ainsi conjointement multiplié les démarches auprès des administrations lozériennes pour faire connaitre leurs revendications. Initiative peu habituelle, elles ont aussi envoyé une lettre d'information aux membres du Comité d'hygiène et de sécurité $(\mathrm{CDH})$ pour exposer leurs réticences et leurs arguments.

«On a réuni des arguments et on a écrit à tous les membres du CDH pour emporter le morceau. J'ai fait un rapport de quelques pages et on leur a envoyé. Mon expérience de professionnel agricole m'a permis de relever des points importants...On leur a suggéré des questions pour le pétitionnaire : les volumes d'eau consommés ne représentaient pas la réalité, par exemple... On est entré dans les contenus techniques du dossier comme la question du substrat karstique »(HERVE, ANPI).

\section{Le rappel à la loi comme épreuve et moyen de faire entendre la parole de nouveaux êtres}

Le 21 décembre 2000, le $\mathrm{CDH}$, dans lequel siègent les principaux services déconcentrés de l'État, des agriculteurs, mais également le délégué d'ANPER-TOS, a voté à bulletin secret un avis défavorable à la demande d'autorisation pour l'extension de l'atelier de truies reproductrices. Très vite, il a été suivi d'un refus de la préfecture. Furieux, les membres de la coopérative ont déféré cette décision devant le tribunal administratif de Montpellier qui avait déjà statué sur l'affaire. Cette fois, la partie requérante n'était plus une association mais les porchers eux-mêmes qui contestaient la décision du préfet. Leur avocat s'est insurgé contre ce qu'il considérait comme un revirement inexplicable de la part des autorités. Il a mis en avant le vote serré du $\mathrm{CDH}$ et la précision de l'étude d'impact que ses clients avaient commandée à un bureau d'étude spécialisé. Celle-ci faisait référence aux risques environnementaux mais proposait aussi des solutions techniques adaptées à la spécificité des lieux, quoique les caractéristiques géologiques mises à jour par les experts du BRGM puissent laisser penser. L'avocat a également mentionné que le réaménagement du site avait conduit à des améliorations. Il a par ailleurs fait référence au plan d'épandage des boues issues de la station d'épuration du Massegros, un bourg voisin, dont personne n'avait jugé bon de s'inquiéter. Selon lui, on ne pouvait pas autoriser d'épandre des boues à l'un alors qu'on interdisait à l'autre d'épandre du lisier.

Les associations ANPER-TOS et ANPI ont apporté leur soutien au préfet visé par ce nouveau procès. Elles ont produit des mémoires en défense communs. Elles se sont principalement attaquées au plan d'épandage fourni par la coopérative porchère, arguant que la capacité épuratrice des sols et la nature des sous-sols étaient incompatibles avec un élevage industriel comme celui de Chanac qui comptabilisait neuf cent quarante six truies, soit en matière de rejets, l'équivalent de deux mille huit cents équivalents porcs, selon un mode de calcul normalisé. S'appuyant sur le rapport du BRGM et reprenant les considérations développées par un des membres fondateurs d'ANPI, elles ont détaillé les caractéristiques géophysiques du Causse de Sauveterre ainsi que ses particularités agronomiques. Elles ont notamment expliqué que les sols karstiques ne filtraient pas les eaux usées, mais aussi que la culture d'une prairie dominée par les légumineuses, comme la luzerne, n'autorisait pas des apports azotés supplémentaires. Les associations ANPER-TOS et ANPI ont cherché à démontrer que sur les deux cent six hectares initialement destinés à l'épandage, cent quatrevingt quatorze hectares étaient beaucoup trop vulnérables. Ce faisant, elles ont jeté un doute 
sérieux sur la valeur des données et des arguments avancés par la coopérative porchère. Elles ont par ailleurs attesté de la relative innocuité des boues minéralisées du Massegros par rapport au lisier.

ANPER-TOS a cité deux cas similaires déjà jugés, un en Bretagne et un dans le Limousin, pour appuyer l'ensemble des moyens qu'elle avait déjà soulevés. Ces précédents ont joué un rôle certain. D'abord en droit, puisqu'ils servent de référence même si la jurisprudence n'a pas en France le poids qu'elle peut avoir dans les systèmes de common $l a w^{39}$. Le précédent indique au juge une ligne de conduite éprouvée et approuvée par ses pairs. Il constitue en outre un réservoir de références et d'arguments dans lequel il est possible de puiser pour donner un sens politique au procès ${ }^{40}$. Dire d'un cas qu'il est identique à un autre ayant marqué les esprits, porte à conséquence. Dans le même temps, cela facilite le travail de problématisation puisque les compétences acquises peuvent être réemployées. Ainsi, après Chanac, ANPI a-t-elle rejoint un groupe d'opposants dans le nord de la Lozère pour contester un autre projet de porcherie industrielle. Les affaires, nous avons pu le voir à maintes reprises lors de notre enquête de terrain, se nourrissent et semblent se contaminer les unes les autres dans l'espace et le temps (les cas bretons, Chanac puis un autre projet de porcherie dans le même département). Elles forment ce que nous avons appelé une casuistique environnementale pour désigner à la fois le travail de liaison entre les cas et ce mouvement d'explicitation, d'articulation et donc de politisation qui fait que des êtres autrefois muets relégués à l'état de contextes indifférents commencent à résister, expriment des exigences, s'imposent collectivement comme des «vivants » avec qui il faut compter ${ }^{41}$. Ainsi compris, l'environnement n'est pas déjà là, donné, mais il se constitue peu à peu par agrégation. Le rural lui non plus n'est pas prédéfini mais se présente sous différentes versions possibles, dont certaines sont plus à même de faire une place aux non-humains, d'accueillir des êtres que les pratiques agricoles intensives tendaient à traiter sans distinction.

Après cinq années de procédure, le second procès s'est achevé par un rejet en 2006. La coopérative porchère a été déboutée de ses demandes et ses projets d'extension ont été désavoués. Elle devra donc continuer à fonctionner sous le régime de la déclaration avec un nombre d'animaux limité. Dans un article de Porc magazine, son avocat s'est insurgé contre la décision du tribunal administratif. Il a fustigé le zèle des services de l'État qui ont usé du pouvoir hiérarchique pour «enliser voire arrêter définitivement un projet» réduisant la Lozère à l'état de «désert porcin ${ }^{42}$. Selon lui, les recherches menées par le BRGM étaient incomplètes et les conclusions qui en ont été tirées étaient hâtives. Il aurait dû y avoir une contre-expertise et le préfet n'aurait pas dû céder aux pressions des environnementalistes. La version du rural défendue par les porchers s'oppose radicalement à celle que l'action contentieuse a permis de faire émerger et d' « énacter.

Malgré l'issue du procès qui leur a été favorable, les associations ANPER-TOS et ANPI restent vigilantes. Elles ont demandé aux autorités de vérifier le nombre d'animaux

\footnotetext{
${ }^{39}$ Serverin, (E.), La sociologie du droit, Paris, La Découverte, 2000.

${ }^{40}$ Chateauraynaud, (F.), Torny, (D.), Les sombres précurseurs. Une sociologie pragmatique de l'alerte et du risque, Paris, EHESS, 1999.

${ }^{41}$ Nous avons repris la notion de casuistique pour décrire ce mouvement de contamination entre les cas et le travail cumulatif d'explicitation des singularités environnementales qui l'accompagne. Pour nous, une fois le travail d'ANPER-TOS accompli, les rivières comme les poissons gagnent en existence et en puissance. Ils deviennent «vivants », si tant est que l'on ne donne pas à ce qualificatif une réalité en soi mais l'envisage plutôt comme un processus au cours duquel des êtres se transforment. Pour une réflexion sur la notion de cas, voir Passeron, (J.-C.), Revel, (J.), « Penser par cas. Raisonner à partir de singularités », Enquête, n 9, 2005, pp.9-44. Pour la notion d'explicitation, on pourra se reporter de manière plus large aux travaux de Peter Sloterdijk.

${ }^{42}$ Les enquêtes de terrain se sont terminées en 2005 avant la fin du second procès. Nous avons mis à jours nos données en recontactant les acteurs qui avaient préalablement accepté de nous rencontrer. Pour un commentaire de la décision de justice par l'avocat des porchers, voir Charles, (C.), «Principe de précaution déguisé. En passant par la Lozère », Porc magazine, complément d'information au n 399, 2006, 5p.
} 
effectivement présents sur le site. Elles ont par ailleurs réclamé que les points de captage des villages environnants soient protégés, s'appuyant sur des analyses ayant révélé la présence de nombreux germes témoins d'une contamination fécale. ANPI avait d'ailleurs fait réaliser une analyse à la fluorescéine qui avait montré que l'eau du Causse s'infiltrait très vite dans le substrat karstique perméable et rejoignait en six heures les principales sources du canton et le Lot, la rivière voisine.

ANPER-TOS (et ses alliés circonstanciels) contribue, par les procès qu'elle intente, à l'explicitation des propriétés des êtres qu'elle représente, mais également à la transformation des états du monde à l'origine de leur dégradation. Les affaires, ne serait-ce que par leur accumulation, produisent de nombreux effets, parfois indirects. Ceux-ci dépassent le simple cadre juridique. Les procès permettent notamment que soient identifiés des êtres menacés par une activité économique ou un projet comme celui de la porcherie de Chanac. Les entités concernées gagnent alors en singularité. Présentées dans un premier temps comme des réceptacles indifférents, les rivières voient leurs débits varier en même temps que leur capacité à diluer les polluants. À Chanac, c'est le Causse qui a révélé ses particularités. Les détails ont proliféré, largement alimentés par les connaissances précises des opposants locaux qui sont venus soutenir l'action contentieuse d'ANPER-TOS. Au fur et à mesure, le sol du Causse de Sauveterre s'est montré karstique et perméable, son sous-sol s'est rempli de failles, la prairie qu'on croyait homogène s'est différenciée, selon qu'il y poussait de la luzerne ou des graminées. Les porcs eux-mêmes se sont scindés en plusieurs groupes, selon que les animaux étaient engraissés ou destinés à la reproduction. Les épandages du lisier se sont distingués des épandages de boues.

Ces différenciations s'accompagnent immanquablement d'un mouvement de liaison. En même temps que les affaires permettent de montrer que de nouveaux êtres comptent, qu'ils doivent être considérés comme des acteurs et non des éléments atones du contexte, elles signalent aussi que chaque masse d'eau doit être pensée comme un réseau de solidarités. L'amont est différent mais ne peut être pensé séparément de l'aval. L'eau s'écoule et redessine sans cesse sa propre géographie. Celle qui s'infiltre à travers la roche poreuse du Causse est susceptible de rejoindre les nappes et la rivière voisine.

Tout le travail d'ANPER-TOS, et des opposants avec qui l'association est amenée à coopérer, est de démontrer que ces paramètres singuliers et les attachements qui les relient n'ont pas été, ou très mal, pris en compte par ses adversaires. Pour cela, elle relance l'enquête à chaque fois, pour chaque être et pour chaque espace concerné de manière à les réassembler. Elle révèle en outre les détails les plus infimes qui font qu'une masse d'eau, qu'un espace, n'est pas substituable à un autre. Elle explique que certains êtres, selon les conditions, n'entretiennent pas entre eux des rapports de composition, comme le lisier avec le karst et une eau favorable à la vie piscicole, à l'alimentation humaine. En tant que porte-parole, elle travaille à démontrer que les non-humains ne doivent pas être traités toute chose égale par ailleurs. Elle contribue surtout à les rendre plus «actifs», plus «vivants ». L'exploration hydrologique engagée par les pêcheurs, couplée avec l'argumentation juridique des professionnels du droit permet de rendre visibles et audibles un nombre plus grand d'êtres à prendre en compte pour la composition des mondes ruraux communs.

Le cas d'ANPER-TOS nous offre une perspective nouvelle sur les manières de concevoir la protestation, c'est-à-dire comme une action collective distribuée entre les humains et les choses. Amateurs de pêche et juristes coopèrent parce qu'ils sont intéressés, les uns par la sauvegarde des salmonidés et les autres par l'application du droit. Les salmonidés, bien qu'ils n'apparaissent pas dans le cas présenté ici, sont des acteurs à part entière de la 
mobilisation puisque ce sont eux qui ont dans un premier temps enrôlé les pêcheurs qui euxmêmes sont allés solliciter des juristes. Ce sont également eux qui ont mobilisé l'ancien président d'ANPER-TOS, membre de la Loutre chanacoise. Les liens inextricables entre les différents acteurs, leurs capacités respectives à se laisser affecter: les poissons par la pollution, les pêcheurs par la disparition des poissons, les juristes par la faible effectivité du droit, en font de bons porte-parole en chaîne. L'articulation de leurs perspectives particulières permet alors de faire des rapprochements inédits, de pointer des problèmes relatifs à des masses d'eau parfois inaccessibles. Les membres d'ANPER-TOS qui sont des porte-parole habilités par le droit repèrent ainsi des êtres nouveaux et suggèrent d'autres façons de faire avec eux. Ce faisant, d'autres modes de relation (de composition et non d'exclusion comme peut en produire l'agriculture intensive) entre ces êtres et d'autres versions de la ruralité sont produites.

Ainsi, on peut dire que faute de mieux, c'est par les affaires que les non-humains sont rendus capables d'objecter aux traitements qu'on leur réserve et accèdent à l'existence politique. Si l'on considère que la sociologie contribue elle aussi à allonger la liste des êtres avec lesquels nous avons partie liée, sans doute doit-on se demander comment -et dans quelles arènes d'accès moins coûteux- elle peut accompagner l'émergence de groupements nouveaux tels qu'ANPER-TOS qui, dans des domaines variés, donnent à entendre des paroles jusque-là inaudibles ${ }^{43}$.

Notice biographique : Christelle Gramaglia est titulaire d'une thèse en sociologie. Elle est chercheure au Cemagref. Elle travaille actuellement sur la genèse de la Directive cadre européenne sur l'eau et la production des indicateurs biologiques pour son application. Elle étudie par ailleurs plusieurs controverses et affaires récentes de pollution (métaux lourds, pesticides et $\mathrm{PCB}$ ).

\footnotetext{
${ }^{43}$ Callon, (M.) «Le renouveau de la question sociale : individus habilités et groupes concernés », Moati, (P.), Nouvelle économie, nouvelles exclusions?, Paris, Aube, 2003 ; Stengers, (I.), La vierge et le neutrino. Les scientifiques dans la tourmente, Les empêcheurs de penser en rond, Paris 2006.
} 